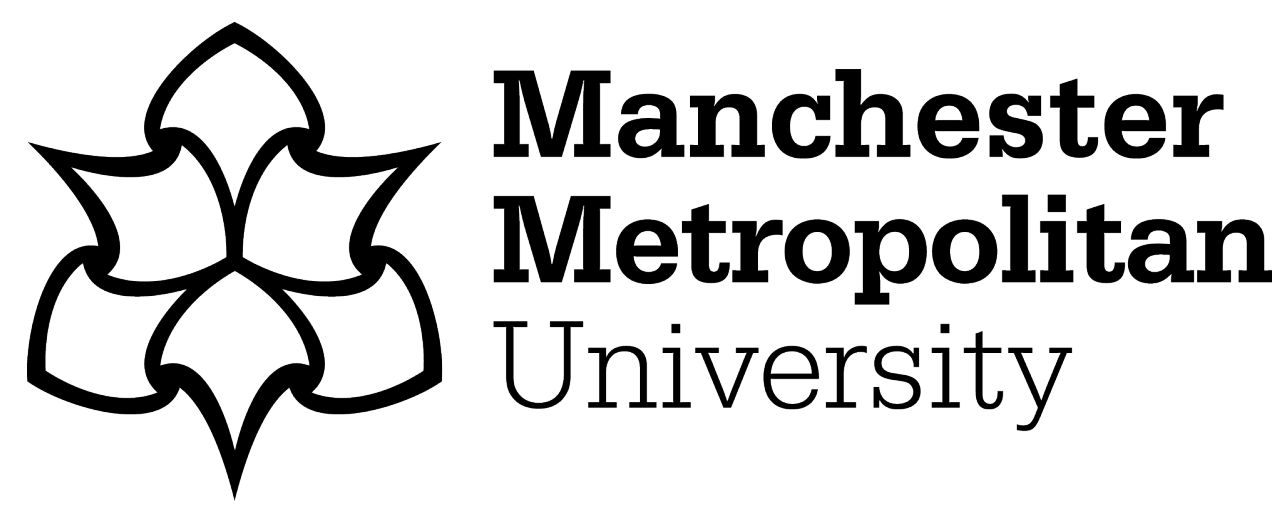

Raja, G, Dhanasekaran, P, Anbalagan, S, Ganapathisubramaniyan, A and Bashir, AK (2020) SDN-enabled traffic alert system for loV in smart cities. In: IEEE Conference on Computer Communications Workshops, 06 July 2020 09 July 2020, Toronto, ON, Canada.

Downloaded from: https://e-space.mmu.ac.uk/628389/

Version: Accepted Version

Publisher: IEEE

DOI: https://doi.org/10.1109/INFOCOMWKSHPS50562.2020.9162888

Please cite the published version 


\title{
SDN-enabled Traffic Alert System for IoV in Smart Cities
}

\author{
${ }^{1}$ Gunasekaran Raja, ${ }^{2}$ Priyanka Dhanasekaran, ${ }^{3}$ Sudha Anbalagan, ${ }^{4}$ Aishwarya Ganapathisubramaniyan \\ ${ }^{5}$ Ali Kashif Bashir \\ ${ }^{1,2,4}$ Department of Computer Technology, Anna University, Chennai \\ ${ }^{3}$ Department of Computer Science and Engineering, SRM Institute of Science and Technology, Chennai \\ ${ }^{5}$ Department of Computing and Mathematics, Manchester Metropolitan University, UK, \\ ${ }^{1}$ dr.r.gunasekaran@ieee.org, ${ }^{2}$ priyankasekard2511@ gmail.com, ${ }^{3}$ sudhaa@ srmist.edu.in, \\ 4aishwarya97.mit@gmail.com, ${ }^{5}$ dr.alikashif.b@ieee.org
}

\begin{abstract}
Intelligent Transportation System (ITS) are helping to enhance road safety and traffic management applications. Internet of Vehicles (IoV) plays a promising role in this field, which turns each vehicle into a smart object with its own compute, storage, and networking capabilities. Nowadays, accidents have been increased mainly due to un-notified alerts about other accidents, work-in-progress, and excessive motorized vehicles at peak times. This non-line of sight information can be efficiently delivered using vehicular communication. IoV network, however has its own challenges like high mobility and dynamic network topology. The above mentioned challenges are addressed with the assistance of a centralized Software Defined Network (SDN), which isolates the control plane from the data plane. In IoV, SDN provides logically centralized traffic management and improves the vehicular communication. In this paper, the Software Defined-Internet of Vehicles (SD-IoV) system is designed to manage heavy traffic and avoids broadcast storm problem with high packet delivery ratio. The proposed broadcast routing mechanism uses selective forwarding and neighbor awareness of the vehicle to efficiently broadcast emergency alert messages, thereby avoiding traffic jams and reducing travel time. On-Board Unit (OBU) in vehicles detects the accident and initializes the broadcast algorithm in SD-IoV system. The accident detection by OBU in vehicles is simulated using machine learning technique with an accuracy of $90 \%$. Simulation performed in SUMO and OMNeT++ shows that with the help of the SDN controller, the IoV network achieves a high packet delivery ratio with minimal delay.
\end{abstract}

Index Terms-IoV, VANET, Vehicle-to-Vehicle, Vehicle-toInfrastructure, Software Defined Network, Broadcast storm.

\section{INTRODUCTION}

According to the World Health Organization (WHO), more than 1.25 million people died because of road accidents. In India, as per 2017 road accident statistics, around 4,64,910 major road accidents occurred due to head-on collision, vehicle turnover, hit from the back, hit from the side, and hitting pedestrians and others. Approximately 20 percent of these accidents had fatal, and on the other hand, the number of road users grows exponentially. IoV Network is capable of delivering various safety and traffic related services to the motorized user and become more attractive to the public.

In IoV, the vehicles that are outfitted with On-Board Unit (OBU) perform efficient communication in Vehicle-to-Vehicle (V2V) communication or Vehicle-to-Infrastructure (V2I) com- munication mode [13]. In-vehicle sensors such as camera, lidar, and radar can capture line-of-sight objects and provide assistance to drivers. The objects or events in the non-line of sight are unable to detect, those information can be delivered by means of vehicular communication. This helps the vehicles to avoid accidents and reduces traffic congestion.

Communicating node uses Dedicated Short Range Communication (DSRC), which provides a $5.9 \mathrm{GHz}$ band with bandwidth of $75 \mathrm{MHz}$ and covers the range of $300 \mathrm{~m}$. The safety application runs in-vehicle detect the accident and also gathers information about the environment. Generally, the traffic or safety-related information gets broadcasted in the network; however, the reason for selective forwarding is that, as vehicles in the opposite direction do not require the information.

In the traditional networking of VANET, the vehicle sends the message to nearby RSU. The RSU sends the message to the control centre present in the ITS, and they forward the message to the respective RSUs. It increases the control overhead in ITS and consumes large bandwidth. Due to the dynamic network topology, the control cost of the network is high. The solution that emerged to lower network control cost is Software Defined Network [9], which simplifies the management and control of the network by isolating the control plane from the data plane. The SDN controller has been aware of global network information using the switches of IoV (RSU \& BS) and interconnection between them to have an optimal routing path. In this paper, the designed SD-IoV system offers the following benefits:

i) The accident detection system in vehicles uses machine techniques to predict the event with an accuracy of $90 \%$

ii) The proposed broadcast routing mechanism make use of selective forwarding and neighbor awareness to efficiently broadcast alert messages, thereby avoids the broadcast storm problem

iii) With the help of SDN controller, alert messages are disseminated with minimal delay and achieves high packet delivery ratio 


\section{RELATED WORK}

IoV is a type of mobile Ad Hoc network especially used for communication between vehicles and RSU and connects the vehicles on the road to form a network.Due to the high mobility of vehicles and dynamic network topology, there is an intermittent connectivity among nodes in the IoV network. The vehicles get location-based services by sharing their location. In greedy based routing, the vehicle sends the packet to a relay node in its range whereas in opportunistic routing, the packet forwarded to the set of relay nodes in its range [4].

The clustering of vehicles in the IoV network helps to improve the message delivery rate and reduces the communication overhead. In clustered IoV network, the cluster head selection can be relied on the low mobility of the vehicle, indeed creating and maintaining the clusters is hard in a dynamic environment In Moving Zone based routing protocol, the periodic update of node location avoided, but it found complicated in cluster maintenance [3]. The peer-to-peer caching is performed to reduce the burden on infrastructure but decreases the cache hit ratio in a highly dense area.

VANET security services becomes mandatory as road users share their location. Autonomous location update and Kanonymity provides privacy for the road users [1]. The AES algorithm establishes a secure beaconing and incorporating the emerging technology of IoT cloud-based system for traffic monitoring delivers the notification through drivers mobile apps [2]. The decentralized traffic monitoring by exchanging segment table [7] is not applicable for high dense areas.

The accident severity can be obtained by sending the video message to the authorities [11]. In [5], the video has been split and transmitted through different independent routes based on the video size but no guarantee of error-free delivery. A costly way of traffic monitoring can be performed by optimizing the patrol vehicle movement in smart city [12].

The SDN-based VANET provides a promising solution to minimize the communication delay and cost [15]. The unicast routing in VANET using SDN with mobility prediction reduces the overall vehicular communication delay [14]. The edge controller and SDN controller for unicast communication had minimized the path failure in the network [6]. SDN based GeoBroadcast mechanism helps to send a periodic broadcast message from the source vehicle to destination location [8]. When a periodic message sent to the same destination, it requires minimal intervention from the controller. By maintaining the flow, the message latency, controller overhead and amount of bandwidth consumed gets reduced, but requires perfect hard time-out [10].

\section{PROPOSED WORK}

In the proposed system, the SDN controller acts as a centralized monitoring system that performs efficient routing based on the global network information obtained from switches. IoV communication is a useful aid in several road safety applications for example, it is used in sending accident alert message to prevent collision.

The system designed to make use of a centralized SDN controller that takes the responsibility of routing the messages,

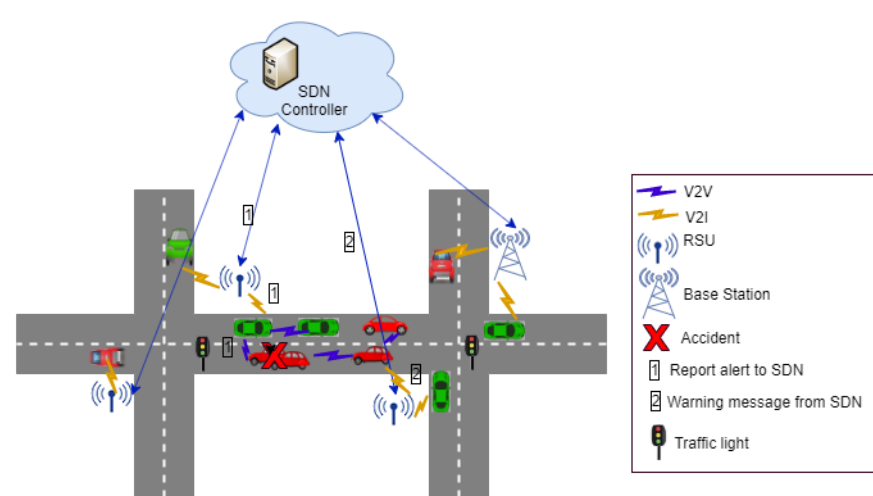

Fig. 1. SD-IoV System Architecture for Alert Message Dissemination

and it cut downs the control cost by separating the control and data plane. The alert message gets broadcasted throughout the network with minimal delay, which has its impact on the reduction of traffic congestion. The in-built capabilities of the SDN controller enable it to maintain a directed graph for the road network and a location component table contains the road segment and their corresponding RSU. In a directed graph of the road network, the junction or intersection represents vertex, and road segments between junctions act as edges. The SDN controller makes use of the directed graph of road network to identify the possible road segment that enters into the accident site. The SDN controller sends the alert message to those RSUs, and the information gets broadcasted to the vehicles in that particular road segment.

The accident information generated by a vehicle in the road segment sent to the SDN controller via nearby RSU as shown in Fig. 1. The vehicle sends the message to the RSU via V2V or V2I communication, which contains vehicle id, RSU id, and Road Segment (RS) id to indicate which road segment gets affected. The SDN controller identifies the possible road segment that enters into the accident site using a directed graph of the road network and then broadcast alerts to it via corresponding RSUs. Thus, the particular lane gets deactivated from the graph until the RSU reports that the route is free from accident. The route-free message from the RSU sent to SDN controller and other RSUs. After receiving the route-free message, SDN activates the affected link, and the RSUs stops broadcasting the alert message to its road segment.

\section{A. IoV Mobility Model}

A model that uses the Poisson process to determine the number of vehicles arrives in a particular segment cannot be utilized here, because of the multiple peak hours available in a smart city. The rate of change is constant in the Poisson process, cannot be applied for today's scenario. The vehicular mobility has been modeled as a NHPP with an arrival rate of $\lambda(t)$ where, number of vehicles arriving at time $t=0, N(0)=$ 0 . The number of vehicles $\mathrm{N}$ varies with time $\mathrm{t}$ is independent. The number of vehicles arrived at time $\Delta t$ is

$$
N(\Delta t)=N(t, t+\Delta t)-N(t)
$$


Probability of no vehicles arriving at time $(t, t+\Delta t)$ is

$$
P\{N(\Delta t)=0\}=1-\lambda(t) \Delta t+o(\Delta t)
$$

Probability of 'n' vehicle arriving at $(t, t+\Delta t)$ is

$$
P\{N(\Delta t)=n\}= \begin{cases}\lambda(t) \Delta t+o(\Delta t) & \text { if, } n=1 \\ o(\Delta t) & \text { if, } n \geq 2\end{cases}
$$

where $o(\Delta t)$ is a negligible function such that

$$
\lim _{\Delta t \rightarrow 0} \frac{o(\Delta t)}{\Delta t}=0
$$

The number of vehicles in road segment $R S_{i d}$ at time $t$ can be characterized as $N\left(R S_{i d}, t\right)$ and density of vehicles is represented as $\rho\left(R S_{i d}, t\right)$. The expectation of the number of vehicles in the road segment $R S_{i d}$ at time $t$ is

$$
E\left(N\left(R S_{i d}, t\right)\right)=\int_{0}^{t} \lambda(x) d x
$$

The probability of exactly $n$ vehicles at time $t$ in $R S_{i d}$ is

$$
P\left\{N\left(R S_{i d}, t\right)=n\right\}=\frac{\left[E\left(N\left(R S_{i d}, t\right)\right)\right]^{n}}{n !} e^{-E\left(N\left(R S_{i d}, t\right)\right)}
$$

The density function depends on the number of vehicles in $R S_{i d}$ at time $t$ is

$$
\rho\left(R S_{i d}, t\right)=\frac{\partial N\left(R S_{i d}, t\right)}{\partial R S_{i d}}
$$

The density of the road segment helps to find whether the road segment is busy or not busy. From RSU, density information is sent to vehicles when they enter the road segment. Based on the information, the vehicles get their minimum threshold speed.

\section{B. IoV System Design}

Assume that, the RSUs are placed at each intersection has index $R S U_{i d}\left(X_{i}, Y_{i}\right)$ and each vehicle has the index $V_{i d}\left(X_{j}, Y_{j}\right)$ where $\mathrm{X}$ and $\mathrm{Y}$ denotes the position of the vehicle in the road segment. The distance covered by the vehicle $D\left(V_{i d}, R S U_{i d}\right)$ in that road segment is the difference between the vehicle index and previous RSU index position, it can be calculated using the Euclidean distance as follows:

$$
D\left(V_{i d}, R S U_{i d}\right)=\sqrt{\left(X_{i}^{2}-X_{j}^{2}\right)+\left(Y_{i}^{2}-Y_{j}^{2}\right)}
$$

Similarly, the interspace distance between the two vehicles in the road segment is also calculated. The speed of the vehicle depends on the density of the road segment $\rho\left(R S_{i d}, t\right)$. The speed of the vehicle $S\left(R S_{i d}, T\right)$ defined as the distance covered by the vehicle at time interval $\mathrm{T}$. The distribution function $f\left(R S_{i d}, S, T\right)$ takes input as road segment, speed $S$ at time $T$ to determine the number of vehicles in that road segment. The mean speed of the vehicle is given by

$$
S\left(R S_{i d}, T\right)=\frac{1}{\rho\left(R S_{i d}, T\right)} \int_{0}^{\max S} S * f\left(R S_{i d}, S, T\right) d S
$$

The acceleration of the vehicle $A c c\left(R S_{i d}, R S U_{i d}, T\right)$ is obtained by rate of change of vehicle speed at time $T$ in a particular direction. It is a vector quantity because it uses the direction of the vehicular movement. The acceleration of the vehicle can be calculated as

$$
\operatorname{Acc}\left(R S_{i d}, R S U_{i d}, T\right)=\frac{S\left(R S_{i d}, T\right)}{d T}
$$

The $R S U_{i d}$ gives the direction of the vehicle by specifying the next-RSU index nearby. The vehicles in the road segment know the location of RSU ahead and traveled road segment RSU. The G-force of the vehicle has been calculated based on the acceleration of the vehicle represented as $\mathrm{Acc}_{x} \mathrm{Acc}_{y}$, $\mathrm{Acc}_{z}$ and gravity as $\mathrm{g}$. The G-force can be calculated as

$$
G-f o r c e=\frac{\sqrt{\left(A c c_{x}^{2}+A c c_{y}^{2}+A c c_{z}^{2}\right.}}{g}
$$

The exposed vibration of a vehicle obtained using G-force value helps to identify whether the accident occurred or not for that vehicle in the road segment. The distance has been calculated based on the information from the neighbor discovery using beacon messages. Each vehicle knows its location using the pre-installed GPS device. The vehicle gives Acknowledgement (ACK) when it receives the beacon message from another vehicle, which contains information like vehicle index and their road segment id. Based on the information, the distance between two nodes (RSU or vehicles) calculated if and only if they present in the same road segment id. The Broadcast Distance Factor (BDF) calculated between the sender and receiver, here the sender denotes the vehicle or RSU, which initiates the beacon message and receiver denotes who gives the ACK message to the sender.

$$
B D F(s, r)= \begin{cases}\frac{D_{s-r}}{R_{v}} & \text { if, } D_{t}>R_{v} \\ \frac{D_{s-r}}{D_{t}} & \text { if, otherwise }\end{cases}
$$

The vehicle which has high BDF is selected to broadcast further in the network. The $R_{v}$ specifies the transmission range of the vehicle, and $D_{t}$ specifies the distance covered by the sender vehicle in that road segment.

\section{Message Transmission}

The vehicles that equipped with OBU detect the accident, whenever there is a sudden change in acceleration and gyroscopic position. The vehicle broadcasts the information to other vehicles and nearby RSU via V2V or V2I communication. The RSU sends the information to the SDN controller, and identifies the road segments which require the alert messages. It also removes the particular affected road segment link (accident site) temporarily from the directed graph. The alert message gets routed to respective RSUs via the rules installed in the switches. The connection among RSUs is known to SDN, and the rule contains a matching field and action field. If the RSU id gets matched, then the action field specifies how to forward packets to reach destination RSU. The received RSU broadcast the message to the vehicles in the road segment at regular intervals. The link of the accident road segment included in the directed graph and other RSUs 
stops broadcasting alert message, when the RSU reports to the SDN and other RSUs that the road segment is clear.

The RSU gets the traffic information from the controller and gives the minimum vehicle speed on the road segment. The density threshold $\left(\rho_{t h}\right)$ of the road segment is defined based on the historical information. The step 1 of algorithm 1, compares the current density with the threshold, if the current density is higher than the threshold, then assign threshold speed as the minimum speed at rush hour else minimum speed at non-rush hour. The minimum threshold speed $\left(V_{t h}\right)$ of the vehicles for that road segment is sent to the vehicle using RSU via direct or indirect V2I communication. In step 5, OBU in vehicle calculates the $A C C_{\text {true }}$, if the speed of the vehicle is less than a threshold, then increment $A C C_{\text {true }}$. If the traffic light signal is GO, then increment the $A C C_{\text {true }}$, if there is a change in acceleration, gyroscopic position of the vehicle, and vibration level of the vehicle. If the $A C C_{\text {true }}$ is greater than or equal to the threshold $A C C_{\text {thres }}$, then the vehicle sends an alert message using the Broadcast_by_vehicle method. If the signal does not turn to $\mathrm{GO}$, then wait for the signal to become GO as per step 16 and then continue the same procedure from step 7 .
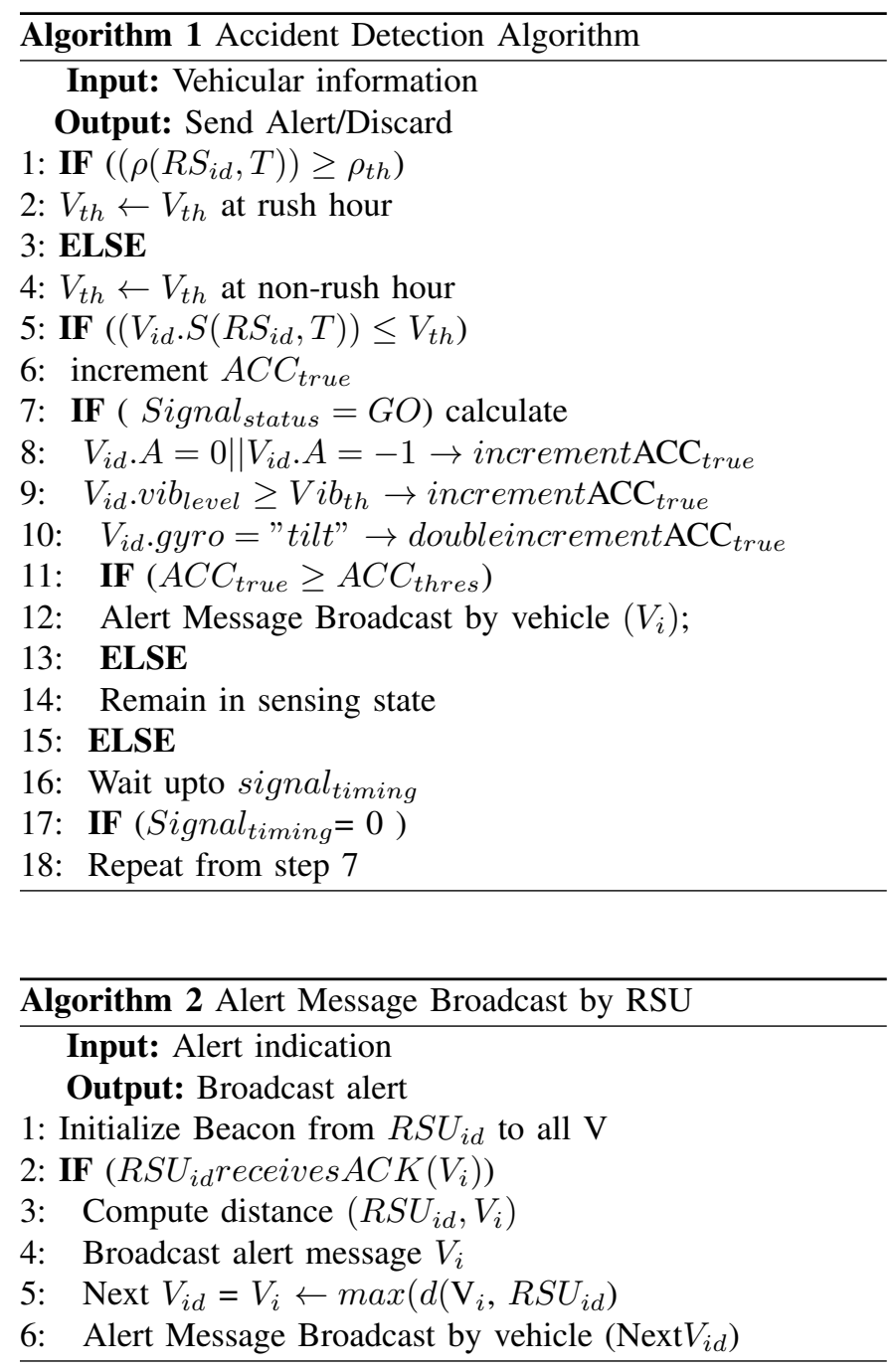

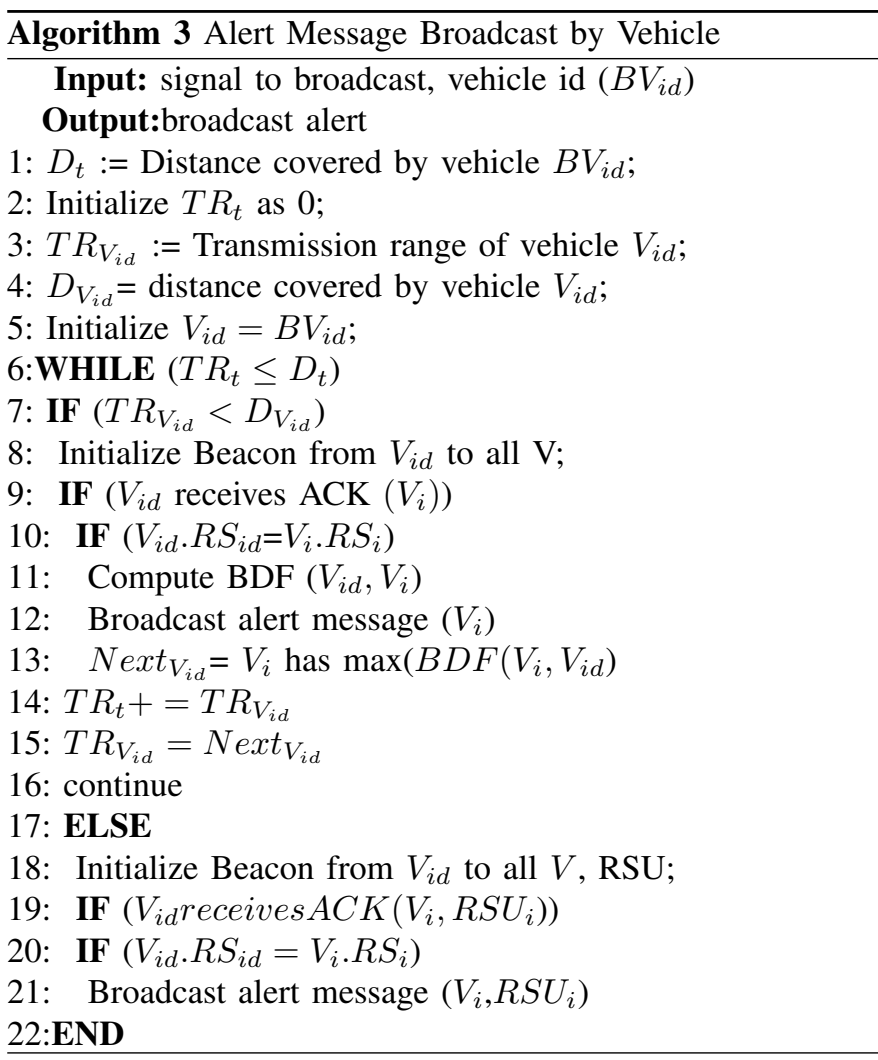

The accident detection algorithm runs in-vehicle detect the accident and sends the alert message to the nearest RSU. As shown in step 1 of algorithm 2, the RSU has to initialize the beacon message to check the neighbor availability in its coverage area, to avoid broadcast storm problem. The RSU has to alert the vehicles in its road segment by efficient broadcast. When the RSU gets a reply message from a vehicle based on the position information of the vehicle, it calculates the distance between them and broadcast an alert message to the vehicles. Step 5, selects the farthest node as next broadcasting node to broadcast further.

The vehicle which detects the accident or has signaled to broadcast from RSU has been termed as broadcasting Vehicle $\left(B V_{i d}\right)$. The broadcasting vehicles calculate the distance covered by the vehicle in that particular road segment $\left(D_{t}\right)$. When the broadcast message reaches the distance $D_{t}$ in that road segment, the selection of next node to broadcast stops. In step 7 of algorithm 3, if the transmission range of the vehicle is less than the distance covered by that vehicle, then the vehicle sends the beacon message to all vehicles in its transmission range. The neighbor vehicle sends the ACK to the source vehicle, by which neighbor's availability is checked. The steps 10 and 20, ensure that both vehicles are in the same road segment, then calculate the distance between the source and neighbor vehicle using a beacon response mechanism. The source vehicle broadcasts the alert message to other vehicles which sent ACK message to it. The next vehicle to broadcast is selected (step 13) based on the distance factor. According to step 14 , the entire transmission range $T R_{t}$ covered is 
incremented by the transmission coverage of the vehicle each time. This procedure continues until the transmission range of the broadcasting vehicle is greater than the distance covered by the vehicle in that segment. Step 17, shown in algorithm 3, if the transmission covered by the vehicle is greater than the distance covered by the vehicle. The source vehicles check the neighbor availability using a beacon message, then broadcast alert message to other vehicles that reply to it.

\section{Performance Analysis}

The vehicle arrival rate considered as an NHPP and different traffic density assumed to evaluate the system performance. Each vehicle has an Event Data Recorder (EDR) to collect information like current location, speed, acceleration, and vibrations exposed. Based on the information in EDR, the vehicle gets its statistical and current data. The malicious user cannot inject the wrong information because the system does not get any input from the user, so the information is valid. The detection of an accident based on information like acceleration, vibration, and speed of the vehicle. The vehicle communicates with RSU (directly or indirectly) and traffic light to get information like minimum speed threshold and signal information, respectively. The accident detection system in vehicles determines whether the vehicle met with an accident or not is closely related to the classification problem, which can be solved using machine learning algorithms. The aim is to classify whether an event occurred or not based on the EDR data and to predict the new instances in real-time.

An ideal system should have high precision and recall to predict the instances correctly. In Fig. 2, the output quality of the various classification algorithm plotted. The considered classifiers are Logistic Regression (LR), Support Vector Machine (SVM), Naïve Bayes (NB), Classification and Regression Tree (CART). The quality can be assessed using accuracy, precision, and recall score of the classifier. But all the classification algorithm is predicting with the accuracy of 90 percent. The EDR data of the vehicle helps to detect the accident accurately.

To predict the current data generated by vehicle as event occurred or not, based on the historic EDR data. The objective of the vehicle is to predict less number of false-negative or false-positive based on the EDR data. In Fig.3, 94 samples of EDR data tested to verify the accuracy of accident detection system in vehicle and results show that there are very few misclassified samples (FP, FN). As shown in Fig. 4, the source

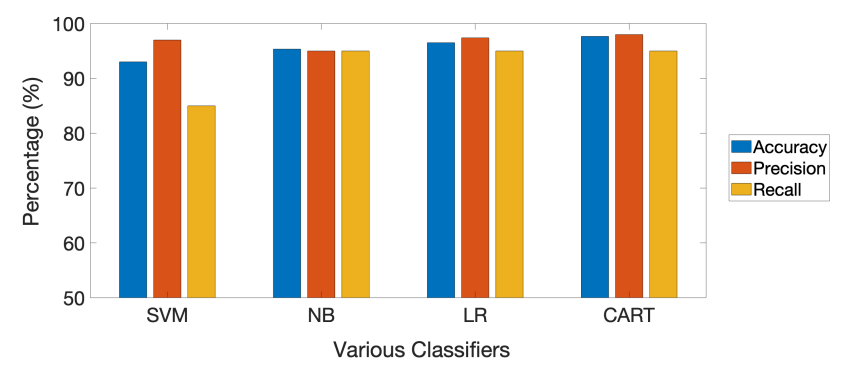

Fig. 2. Performance Comparison of various classifiers

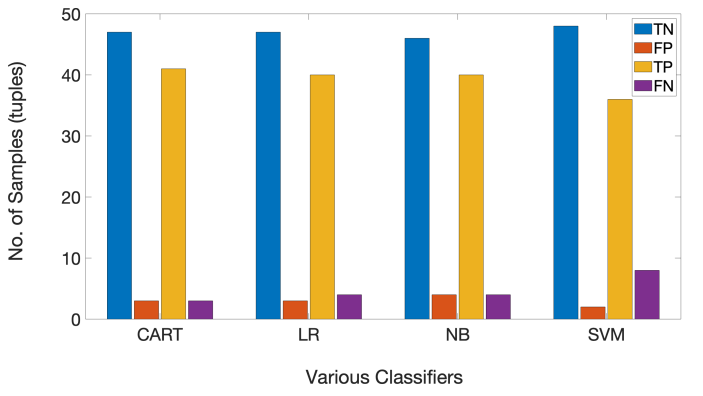

Fig. 3. Number of samples vs Classifiers

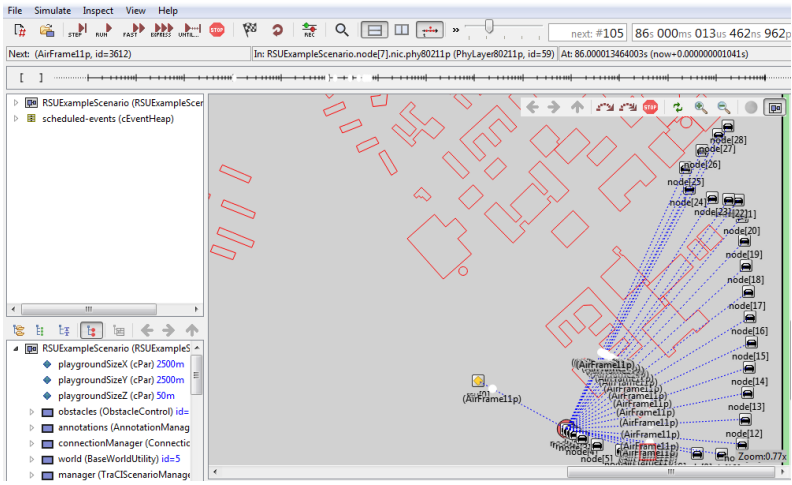

Fig. 4. Alert Message Dissemination and Forwarder Selection

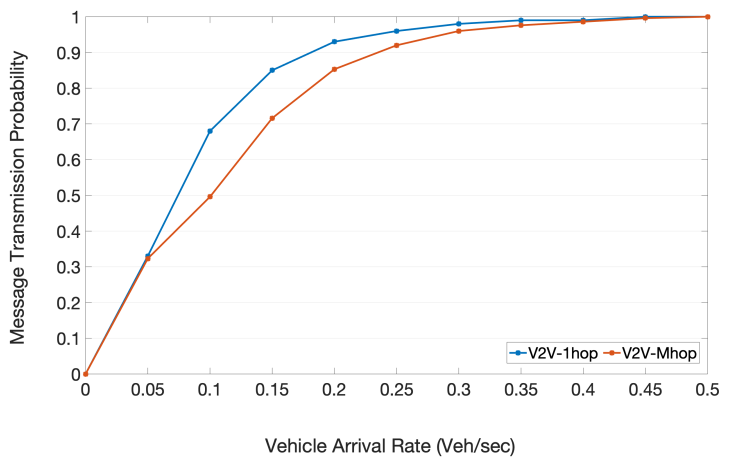

Fig. 5. V2V n-hop message transmission probability

vehicle which met with an accident broadcast the alert message and also selected the next forwarder vehicle to broadcast the message further. The vehicle represents nodes in OMNet. Veins help to integrate SUMO and OMNet. The message transmission from the vehicles to RSU or other vehicles as depicted. The simulation parameters, as shown in Table 1. The successful message transmission probability increases with increasing vehicle arrival rate. In Fig. 5, increasing the hop count in V2V communication, actually decreases the probability of message transmission. The propagation speed increases with an increase in vehicle density on the road segment, as shown in Fig.6. The speed increases because the next forwarder vehicle is farthest from the broadcasting node range. 
An increase in vehicle density on the road decreases the speed of the vehicles in that segment. From the simulation results of machine learning shows that the vehicle can accurately predict the new instance obtained in real-time and broadcast the alert message to nearby RSU and vehicles. The simulation results obtained from OMNeT ++ show that an increase in vehicle density achieves a high probability of message transmission and message propagation speed.

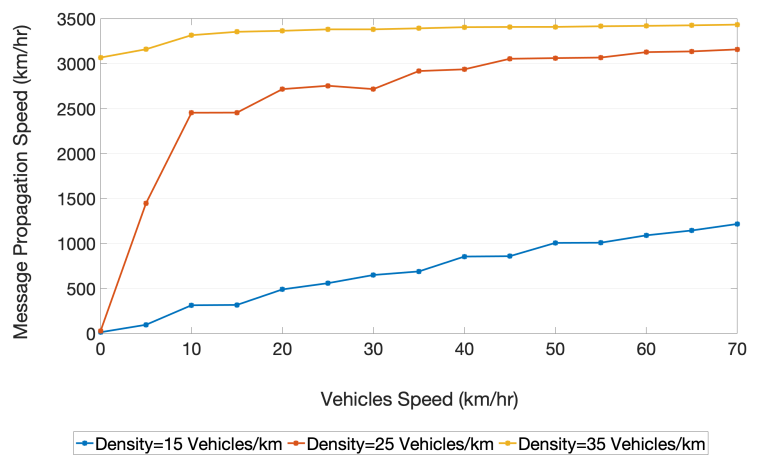

Fig. 6. Impact of Vehicle Speed on Message Propagation Speed

\section{CONCLUSION}

In our current work, the RSU has been placed at the intersection and assumed that no failure in RSU. To avoid congestion, road segment users require traffic information about their travel path. With the help of the SDN Controller, road users get traffic information about other road segments too. The supervised classifier used to predict whether an accident happened or not for a particular vehicle using the information like acceleration force, gyroscope position, and vibration level it exposed. The On-Board Unit in each vehicle runs this algorithm whenever there is a change in acceleration than the threshold limit. If the accident occurred, it sends the alert message to the surrounding vehicles and nearby RSU using the proposed broadcast algorithm. This system reduces traffic congestion and travel time. Thus, with the help of vehicular communication, the drivers get the non-line of sight information about road conditions. In the future, a plan for optimal placement of RSUs efficiently, to reduce the overall cost of the system and to handle the failure of RSUs if it occurs.

TABLE I

SimUlation PARAMETERS

\begin{tabular}{|c|c|}
\hline Parameter & Value \\
\hline V2V Communication protocol & DSRC \\
\hline Mobility Model & NHPP \\
\hline Transmission range of vehicle & $200 \mathrm{~m}$ \\
\hline Vehicle transmission power & $10 \mathrm{dBm}$ \\
\hline Bandwidth & $10 \mathrm{MHz}$ \\
\hline
\end{tabular}

\section{ACKNOWLEDGEMENT}

This Publication is an outcome of the R\&D work undertaken in the project under the Visvesvaraya PhD Scheme of Ministry of Electronics Information Technology, Government of India, being implemented by Digital India Corporation (formerly Media Lab Asia).

\section{REFERENCES}

[1] Belal Amro, Yucel Saygin, and Albert Levi, "Enhancing privacy in collaborative traffic-monitoring systems using autonomous location update", IEEE Transactions on Intelligent Transportation Systems, Vol. 7, No. 4, pp. 388-395, 2013.

[2] Celesti, Antonio Galletta, Lorenzo Carnevale, Maria Fazio, Aine LayEkuakille, and Massimo Villari, "An IoT Cloud System for Traffic Monitoring and Vehicular Accidents Prevention Based on Mobile Sensor Data Processing", IEEE Sensors Journal, Vol. 18, No. 12, pp. 4795-4802, 2017.

[3] Dan Lin, Jian Kang, Anna Squicciarini, Yingjie Wu, Sashi Gurung, and Ozan Tonguz, "MoZo: A Moving Zone Based Routing Protocol Using Pure V2V Communication in VANETs", IEEE Transactions on Mobile Computing, Vol. 16, No. 5, pp. 1357-1370, 2016.

[4] Li Ning, Martinez-Ortega Jose-Fernan, Hernandez Vicente, and Fernandez Jose, "Probability Prediction based Reliable Opportunistic (PRO) Routing Algorithm for VANETs", IEEE/ACM Transactions on Networking, Vol. 26, pp. 1933-1947, 2017.

[5] Mostafa Asgharpoor Salkuyeh, and Bahman Abolhassani, "An adaptive multipath geographic routing for video transmission in urban VANETs", IEEE Transactions on Intelligent Transportation System, Vol. 17, No. 10, pp. 2822-2831, 2016.

[6] Muhammad Tahir Abbas, Afaq Muhammad, and Wang-Cheol Song, "SD-IoV: SDN enabled routing for internet of vehicles in road-aware approach", Journal of Ambient Intelligence and Humanized Computing, pp. 1-16, 2019.

[7] Thales Teixeira de Almeida, Jose Augusto Nacif, Fabiano Pereira Bhering and Jose Geraldo Ribeiro Junior, "DOCTraMS: A Decentralized and Offline Community-Based Traffic Monitoring System", IEEE Transactions on Intelligent Transportation Systems, Vol. 20, No. 3, pp. 1160-1169, 2018.

[8] Yu-Chun Liu, Chien Chen, and Suchandra Chakraborty, "A Software Defined Network architecture for GeoBroadcast in VANETs", IEEE International Conference on Communications (ICC), pp. 6559-6564, 2015.

[9] Sudha Anbalagan, Dhananjay Kumar, Gunasekaran Raja, Alkondan Balaji, "SDN assisted Stackelberg Game model for LTE-WiFi offloading in 5G networks", Elsevier - Digital Communication and Networks, Vol. 5, No. 4, pp. 268-275, 2019.

[10] Sudha Anbalagan, Dhananjay Kumar, Mercy Faustina J, Gunasekaran Raja, Waleed Ejaz, Ali Kashif Bashir, "SDNAssisted Efficient LTE-WiFi Aggregation in Next Generation IoT Networks", Elsevier - Future Generation Computer Systems, https://doi.org/10.1016/j.future.2017.12.013, 2017.

[11] Gunasekaran Raja, Anil Thomas, "SAFER: Crowdsourcing based Disaster Monitoring System using Software Defined Fog Computing", Springer - Wireless Networks, Vol. 24, N0. 5, pp. 1414-1424, 2019.

[12] Anil Thomas, Gunasekaran Raja, "FINDER: A D2D based Critical Communications Framework for Disaster Management in 5G", Springer-Peer-Peer Networking and Applications, Vo. 12, No. 4, pp. 912-923, 2018.

[13] Gunasekaran Raja, Aishwarya Ganapathisubramaniyan, Sudha Anbalagan, Sheeba Backia Marry Bhaskaran, Kathiroli Raja, Ali Kashif Bashir, "Intelligent Reward based Data Offloading in Next Generation Vehicular Networks", IEEE Internet of Things Journal, DOI:10.1109/JIOT.2020.2974631, 2020.

[14] Rajesh Gupta, Sudeep Tanwar, Sudhanshu Tyagi, Neeraj Kumar, "Machine Learning Models for Secure Data Analytics: A taxonomy and threat model, Computer Communications", Vol. 153, pp. 406-440, 2020.

[15] Zhihan Lv, Neeraj Kumar, "Software defined solutions for sensors in 6G/IoE", Computer Communications, Vol. 153, pp. 42-47, 2020. 